\title{
Update to the methodology used to calculate health expectancies for the UK and constituent countries
}

Michael P Smith, Olugbenga Olatunde and Chris White Office for National Statistics

\section{Abstract}

\section{Background}

Changes to the design of the General Household Survey (GHS) risk a discontinuity in the ONS Health Expectancy (HE) series. This report compares methodological approaches to ensure consistency of this important National Statistic and reports improvements to the methodology surrounding standard error (SE) estimation.

\section{Methods}

A comparison of the use of cross-sectional and longitudinal GHS data in estimating health state prevalence and HE in the UK, GB and constituent countries for the period 2005-07. Incorporation of the GHS sampling design effect into the calculation of standard errors surrounding estimates of HE backdated to 2000-02.

\section{Results}

The prevalence of favourable health states was significantly higher in the longitudinal compared to the cross-sectional sample of the GHS. Compared to established trends, the inclusion of longitudinal data in estimates of healthy life expectancy (HLE) led to implausible increases in this metric. However, the prevalence of limiting long-standing illness and estimates of disability-free life expectancy (DFLE) using longitudinal data were broadly comparable and in line with recent trends.

Inclusion of the design effect had only a minor impact on the estimation of current and historic HE standard errors. On three occasions, increases in HLE over time were no longer significant using this enhanced method of SE estimation and in a single instance differences in DFLE between countries were no longer significant.

\section{Conclusions}

Attrition in the GHS longitudinal dataset introduces significant bias into estimates of health state prevalence and a discontinuity in the established trend of HLE. Utilising the crosssectional GHS sample alone in 2005-07 represents the most reliable approach to maintain the consistency of this important series. Incorporation of the design effect enhances the precision of estimates of HE. 


\section{Contents}

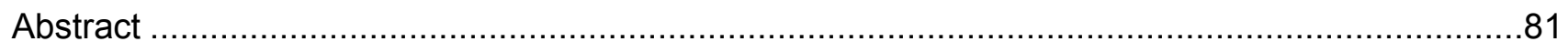

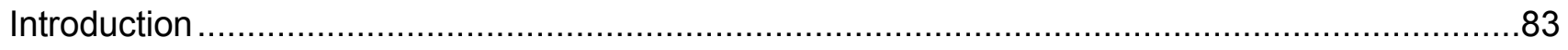

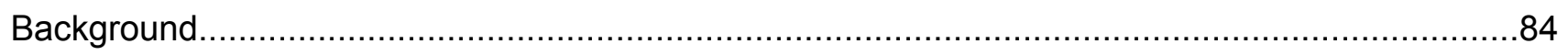

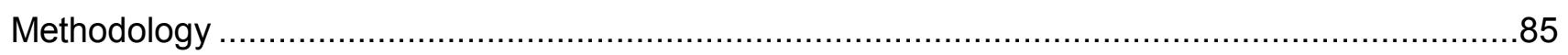

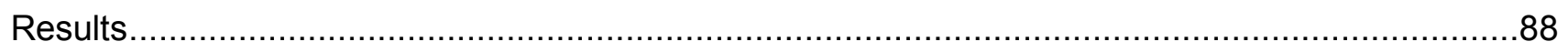

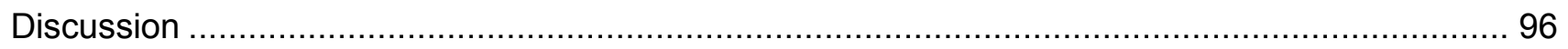

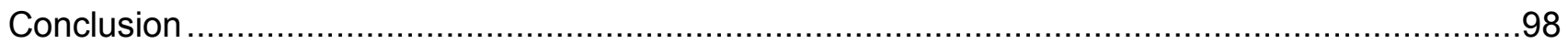

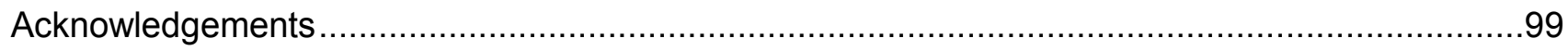

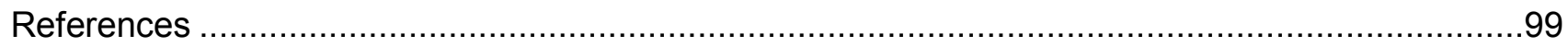

\section{List of Tables}

Table 1 The prevalence of 'Good'/'Fairly Good' health and limiting long-standing illness for males and females by GHS(L) wave in 2005, 2006 and 2007 ....

Table 2 The prevalence of 'Good'/'Fairly Good' health and limiting long-standing illness by sex in complete and cross-sectional specific GHS(L) datasets 2005-2007 ....

Table 3 HLE and DFLE by complete and cross-sectional specific survey data at birth and at age 65 by sex: UK, GB and constituent countries, 2005-2007

Table 4 Comparison of average change in HLE over time with estimates derived from complete and cross-sectional specific datasets

Table 5 Average precision of estimates of HLE and DFLE at birth and at age 65 by sex in original and deff adjusted 95 per cent $\mathrm{Cl}$ : UK, GB and constituent countries 20002002 to $2004-2006$ 


\section{List of Figures}

Figure 1 HLE for males at birth: by complete and cross-sectional specific data 2000-2002 to

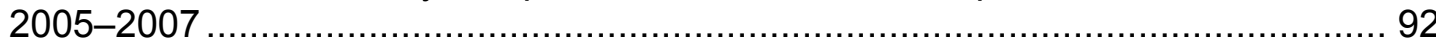

Figure 2 HLE for females at birth: by complete and cross-sectional specific data 2000-2002

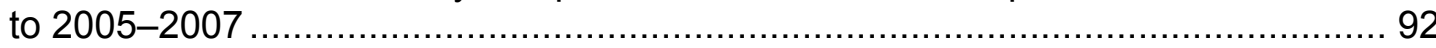

Figure 3 HLE for males at age 65: by complete and cross-sectional specific data 2000-2002 to $2005-2007$

Figure $4 \quad$ HLE for females at age 65: by complete and cross-sectional specific data 20002002 to $2005-2007$

\section{Introduction}

In 2005 the design of the General Household Survey (GHS) changed to a rotating panel, to give researchers access to data capable of measuring changes over time in respondents' circumstances, including their states of health. The advantages for research of longitudinal data have been well documented; ${ }^{1}$ however, a weakness is the possibility of loss to follow-up among sample members. This can introduce bias into analyses if the cause of the attrition is related to the subject under study, such as well-being or functional health status.

The introduction of longitudinal data capture into the GHS has implications for the continuity of the Health Expectancy (HE) time series which has been published by the Office for National Statistics (ONS) since 1981. Disproportionate attrition of a particular group of contributors, for example those in the poorest health, will preclude the use of repeat measurement data (panels two to four) in maintaining the time series. However, restricting the analysis to the annual refreshed sample (panel one only) may result in insufficient numbers to detect statistically significant differences between constituent countries of the UK and over time.

This report has three principal aims:

- Firstly, to compare the prevalence of health states in the longitudinal and cross-sectional elements of the GHS, in order to assess the extent of bias resulting from attrition

- Secondly, to compare HEs derived from survey data that include and exclude the longitudinal elements of the GHS, in order to inform the likely effect of using the longitudinal data in this time series

- Thirdly, to document the approach to calculation of the standard errors (SE) of HE estimates incorporating the survey design effect (deff). This approach is applied to the historical time series from 2000-02, to ensure that the variance of health expectancy estimates and their precision take account of the complex sampling design of the GHS 


\section{Background}

ONS estimates of HE for the UK and its constituent countries are partly constructed using the selfreported health of respondents to the GHS. In 2005, this survey changed from a purely crosssectional design to a rotating-panel longitudinal design, becoming the General Household Survey Longitudinal, GHS(L). In the same year, the survey sample was arbitrarily divided into four distinct panels, each being representative of the wider private household population of Great Britain (GB).

Three of the panels interviewed in 2005 were interviewed again in 2006, representing the first round of longitudinal or repeat-survey data. A single panel (wave 1 in 2006), was recruited afresh, providing cross-sectional data in that year. In 2007, three panels from the previous year were again carried forward for interview, representing the second (wave 2) or third (waves 3 and 4) instalments of longitudinal data. Again in 2007, a single panel (wave 1 in 2007) was recruited to interview for the first time, providing cross-sectional data for that year.

Although not included in the analysis presented here, 2008 will be the first year in which a panel in the $\mathrm{GHS}(\mathrm{L})$ will have been interviewed on four successive occasions. Another novelty in 2008 is the presence of panels in their first, second, third and final year of interview (Box 1). Note that in 2008 the GHS $(\mathrm{L})$ was a constituent part of the Integrated Household Survey (IHS) becoming the General Lifestyle Module (GLF).

In each year from 2006, the new survey entrants (wave 1) represented approximately one-third of the total survey sample. This was to over-compensate for potential attrition in the panel as it underwent re-interview in successive years. The new (wave 1) panel in each year represents cross-sectional data and as such does not suffer from potential bias that may arise through attrition. This change in the design of the data source used by ONS to calculate HE, places at risk the continuity of this time series, established in 1981 for GB and in 2001 for the UK as a whole.

The standard approach to calculating HEs involves pooling three consecutive years of survey data in order to increase sample sizes and achieve sufficient precision to identify differences between countries and changes over time. With the introduction of longitudinal data elements, simply repeating the pooling of three years survey data would involve using repeat observations. The use of repeat observations has certain implications including bias that may arise from panel conditioning and changes in the population that occur between the time of selection of the panel and interview. ${ }^{1}$

The key aspect of concern here is the possibility of non-random attrition: that is, disproportionate loss from the sample of particular sub-groups of the sample, such as those in the poorest health. In the case of HEs, this will exaggerate the length and proportion of life that someone might expect to live in 'Good' or 'Fairly Good' health or without a limiting chronic illness. Sample weights have been developed for the GHS(L) to correct for non-random attrition, but there is no guarantee that these weights fully rectify the effect of attrition on the outcome under study.

Another consequence of using repeat observations is a reduction in the precision of calculated estimates, which arises as a result of inflation in the SEs caused by the correlation in within-subject responses over time. 
An alternative to pooling the full datasets is to extract only the new entrant panels for pooling over time. This approach was used for HE estimates reported in 2004-2006. As the observations are cross-sectional, the issues arising with longitudinal responses are eliminated. However, the available sample for analysis is substantially smaller than was the case prior to 2006. This has two potential consequences: firstly, there may be no observations in some of the cells required for the $\mathrm{HE}$ tables; secondly, as the SE is a function of the sample size, the level of precision in $\mathrm{HE}$ estimates will reduce.

This article reports preliminary findings arising from an exploration of including or excluding the longitudinal component of the GHS(L) in calculating HEs in the period 2005-2007. The primary focus is the effect on consistency. Further work is being carried out on the effect on precision of the HE estimates, which will be reported at a later date. In addition, this article also reports improvements made to the standard method of sampling error estimation, backdated to the period 2000-2002.

\section{Methodology}

ONS estimates of HE are calculated using the Sullivan method, which requires the combination of survey, mortality and population data to estimate periods of life spent in 'Good' or 'Fairly Good' health; healthy life expectancy (HLE), or without a limiting chronic illness or disability; disability-free life expectancy (DFLE). ${ }^{2}$

\section{Health state prevalence}

A primary component of HE estimation is the population prevalence of health states by sex and five-year age band. For GB and constituent countries, these are calculated from responses to general health and limiting long-standing illness questions in the GHS(L). For the UK, GHS(L) data is combined with responses to identical questions in the Continuous Household Survey (CHS) of Northern Ireland (NI).

GHS $(L)$ survey data is weighted to compensate for non-response and calibrated to the sampling distribution of known population totals in terms of age group, sex and region. This weighting allows the survey data to reflect the population characteristics of GB and lower level geographies. In 2006, weighting was adjusted to include non-response due to attrition, that is, non-participation of respondents to subsequent waves of the survey. For the CHS a simple, population based, weighting is applied to survey data to reflect the mid-year population estimates of NI by age and sex.

Weighted survey data is used to calculate population estimates of the prevalence of 'Good' or 'Fairly Good' and 'Not Good' health states and the presence or absence of a limiting long-standing illness which feed into the construction of the HE metrics. This is combined with prevalence estimates of residents of medical and care communal establishments, based on the prevalence rates recorded at the Census 2001 and adjusted to match current mid-year population estimates. It is necessary to use the 2001 Census data to estimate the prevalence of health states in the communal establishment population since the scope of $\mathrm{GHS}(\mathrm{L})$ and $\mathrm{CHS}$ data is restricted to the private household population. 


\section{Health expectancies}

The complete methodology of ONS HE estimation has previously been published and will not be described in detail here. ${ }^{3}$ Briefly, three-year aggregate survey and communal establishment health prevalence data is multiplied by the total person years lived at a given age interval, calculated from three-year aggregated mid-year population estimates and period life tables, to give the total number of person years lived in that age-interval in 'Good' or 'Fairly Good' health or without a limiting long-standing illness. This value is then divided by the number of people surviving to that age to give an estimate of HLE or DFLE respectively.

\section{Design of the GHS(L)}

As described earlier, households surveyed by the GHS in 2005 were arbitrarily divided into one of four 'waves' to indicate their position in the rotating panel of subsequent $\mathrm{GHS}(\mathrm{L})$ surveys. In each year from 2006, wave 1 respondents represent the new and therefore cross-sectional element of the survey, while waves 2 to 4 represent repeat entrants (Box 1).

\section{Box 1 Diagrammatic representation of waves through successive years of the GHS(L) (General Lifestyle Module from 2008)}

\begin{tabular}{|c|c|c|c|}
\hline Wave 4 & & & \\
\hline Wave 3 & Wave 4 & & \\
\hline Wave 2 & Wave 3 & Wave 4 & \\
\hline Wave 1 & Wave 2 & Wave 3 & Wave 4 \\
\hline \multirow[t]{4}{*}{2005} & Wave 1 & Wave 2 & Wave 3 \\
\hline & 2006 & Wave 1 & Wave 2 \\
\hline & & \multirow[t]{2}{*}{2007} & Wave 1 \\
\hline & & & 2008 \\
\hline
\end{tabular}

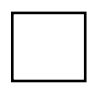

New entrants (cross sectional)

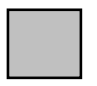

3rd year of survey (longitudinal)

2nd year of survey (longitudinal) 4th year of survey (longitudinal)

\section{Comparison of longitudinal and cross-sectional data}

The purpose of the first investigation was to establish the extent to which the attrition weighting was compensating for differential attrition. The assumption was that in any year after 2005 the health state prevalence estimates from the new entrant (wave 1) panel should not be significantly different from the corresponding estimates from the continuing (waves 2 to 4 ) panels. Appropriate weights were applied to estimates from each panel. This is because each panel was selected as a representative sample of the population. The ongoing panels exclude exits from the cross-sectional 
population caused by deaths and ineligibility; but do not account fully for entrants to the crosssectional population.

Any differences that do occur in estimates between the new and ongoing panels are therefore attributed to the longitudinal process, that is, attrition, population change or panel conditioning. It has been assumed that imperfect adjustment for attrition will be the major cause of any such differences, although it is not possible to verify this with certainty.

Health state prevalence was compared between wave 1 (cross-sectional) and waves 2 to 4 (longitudinal) in 2005, 2006 and 2007. Both weighted and unweighted data were compared in each period, since the addition of a model to account for population based attrition in the weighting paradigm of the GHS $(\mathrm{L})$ might account for bias caused by the loss of survey respondents in the longitudinal data.

HLE and DFLE by country, sex and age band based upon complete GHS(L) datasets over the period 2005-2007, that is, incorporating both the cross-sectional and longitudinal elements, were compared with the cross-sectional data alone over this period.

At the time of writing, the standard errors of the complete dataset had yet to be calculated. These require the estimation of the over-time correlation between longitudinal responses, for which the methodology is being developed. This will then enable statistical significance tests to be undertaken on differences between the complete and cross-sectional data.

\section{Calculation of confidence intervals}

ONS estimates of HLE and DFLE are reported with 95 per cent confidence intervals (Cls) to indicate their precision. The 95 per cent $\mathrm{Cl}$ is additionally used to detect significant differences between countries and over time. Historically, the SE and by extension, the Cls, have been calculated for both the GHS(L) and the CHS without taking into account their survey design. This approach is appropriate for surveys with a simple random sample design, such as the CHS. However, for surveys with complex sample designs, such as the GHS(L)'s cluster design, a more accurate estimation of SEs is necessary, incorporating the deff of sample selection.

The GHS(L) has a multi-stage sample design, involving clustering and stratification, each of which has different effects on SEs. For example, if health status among people living within the same primary sampling unit (PSU) is more similar than it is among the population in general, the standard errors of HE estimates can increase. In contrast, stratification results in reduced SEs and therefore better precision of point estimates.

These deffs should be accounted for when calculating the SEs of HE estimates since the size of the error depends upon the spread of health events within and between PSUs. ONS HE timeseries backdated to 2000-2002 has been adjusted in this paper to reflect the deff of the GHS over these years, and these data are compared with original estimates of 95 per cent Cls. Estimates for $\mathrm{NI}$ are unaffected by this issue since the CHS has a simple random sample design. However for the UK, SEs from GB incorporating the deff of the GHS has been combined with those of $\mathrm{NI}$ to calculate UK level SEs and 95 per cent Cls. 


\section{Results}

\section{Comparison of the prevalence of health states between cross-sectional and longitudinal data}

The analysis reported below demonstrates that the prevalence estimates of health states for those people continuing in the survey beyond the first (wave 1) interview are different from those entering the survey for the first time. In the unweighted data, 'Good' and 'Fairly Good' health was significantly more prevalent in longitudinal (waves 2 to 4 ) compared with cross-sectional (wave 1) data in 2006 and 2007. There was no statistically significant difference between the waves in 2005, when all respondents were cross-sectional. In addition, there were no significant differences in the prevalence of limiting long-standing illness between longitudinal and cross-sectional panels for males or females in 2006 and 2007 or between panels in 2005 (see Table 1).

In 2006, 91.2 per cent of males in the longitudinal panels reported 'Good' or 'Fairly Good' health compared with 89.3 per cent in the cross-sectional panel. Similarly in 2007 , the proportion of males reporting favourable health states in the longitudinal panels was significantly greater than in the cross-sectional panel (90.9 per cent and 89.4 per cent respectively). For females in 2006, 90.2 per cent in the longitudinal panels reported 'Good' or 'Fairly Good' health compared with 86.9 per cent in the cross-sectional panel. In 2007 these figures were 89.6 per cent and 87.8 per cent respectively.

When weighted to reflect non-response and attrition, the prevalence of 'Good' and 'Fairly Good' health was significantly higher in the longitudinal compared with the cross-sectional panels in 2006 but not in 2007. For males in 2006, 91.5 per cent reported favourable health states in the longitudinal panels compared with 89.4 per cent in the cross-sectional panel. In 2007 these figures were 91.1 per cent and 89.7 per cent respectively. For females in 2006, 90.0 per cent reported 'Good' or 'Fairly Good' health in the longitudinal panels compared with 86.7 per cent in the crosssectional panel. In 2007 these figures were 89.4 per cent and 87.8 per cent respectively. There were no differences in the weighted data for health state prevalence between panels in 2005 and in the prevalence of limiting long-standing illness between longitudinal and cross-sectional panels in 2005, 2006 and 2007. 


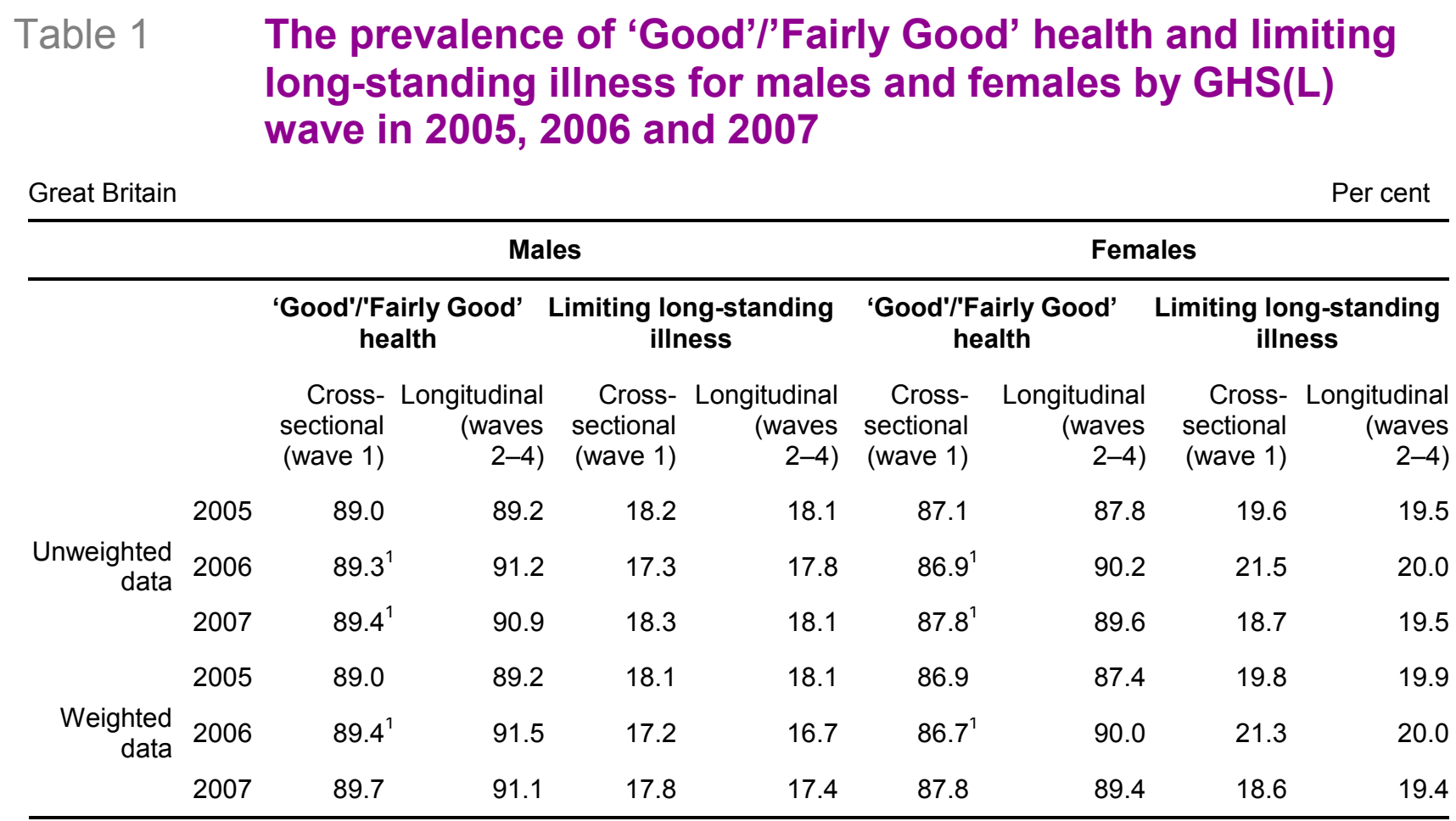

${ }^{1}$ Significant difference between waves $(p>0.05)$

\section{Health expectancies}

Survey data were combined to calculate HEs for the period 2005-2007. Comparisons were made between complete (waves 1 to 4 in each year) and cross-sectional datasets (comprising waves 1 to 4 in 2005 and wave 1 only in 2006 and 2007).

As with the comparison between longitudinal and cross-sectional panels, 'Good' and 'Fairly Good' health was more prevalent for males and females in the complete dataset compared with the cross-sectional data alone. For males the prevalence of favourable health states was 90.0 per cent in the complete dataset compared with 89.2 per cent in the cross-sectional data, for females the equivalent figures were 88.5 per cent and 87.5 per cent respectively. There were mostly no differences in the prevalence of limiting long-standing illness between complete and crosssectional datasets (Table 2). 


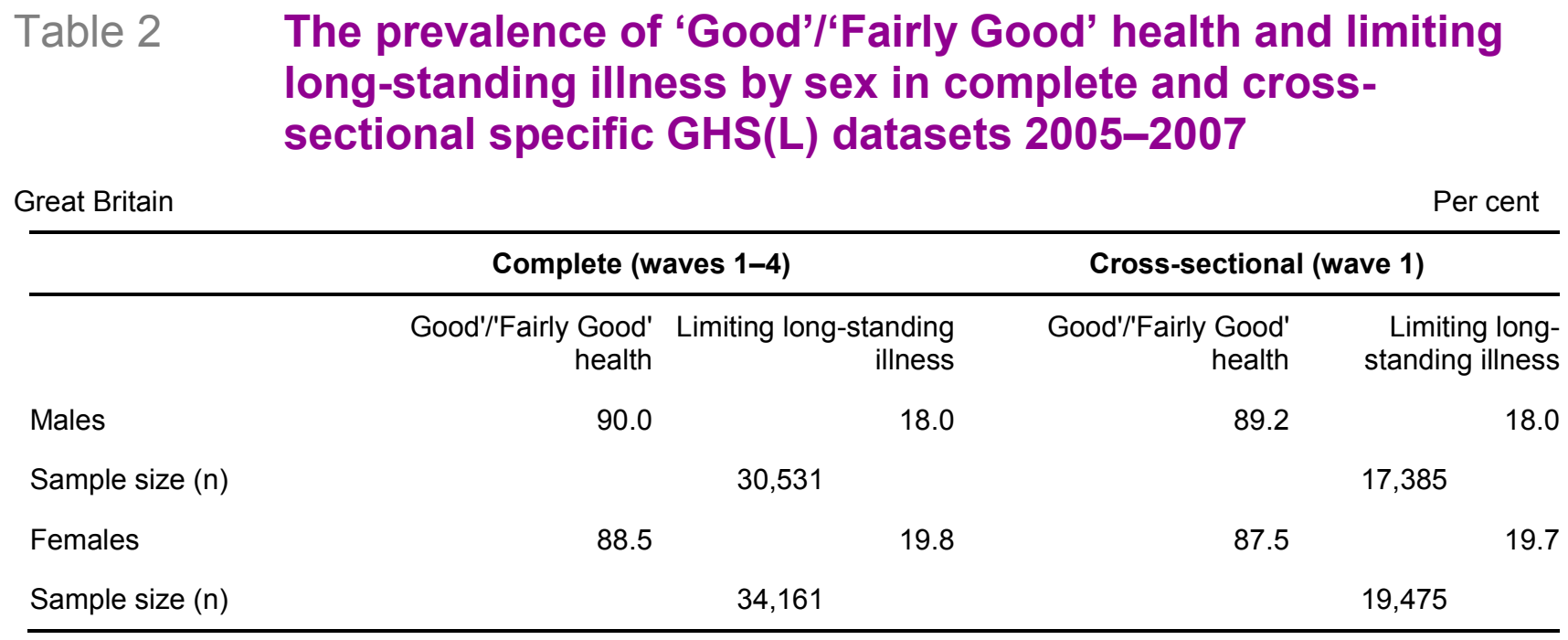

Complete and cross-sectional datasets from the $\mathrm{GHS}(\mathrm{L})$ were combined with data from the $\mathrm{CHS}$, mid-year population estimates, and mortality data to calculate health expectancies for the UK, GB and constituent countries in 2005-2007.

Although it is not possible here to assign statistical significance to differences between HE estimates derived from complete and cross-sectional data, clear patterns in the data emerged.

In general, HLEs for males and females at birth and at age 65 were higher when derived using the complete data. The greatest disparity was in males at birth in the UK, England and Scotland where HLE derived from complete data was 0.8 years greater than that derived from the cross-sectional specific data. For females the greatest disparity occurred in Wales at birth, where the complete data produced an estimate 1.5 years higher than that estimated using cross-sectional data (Table 3).

There was a single instance in which the HLE estimates were identical; males in Scotland at age 65. There was also one instance in which the estimate derived from the complete data was lower than that produced by the cross-sectional data; males in Wales at age 65 had an estimated HLE of 12.3 years using to the complete data and 12.7 years using the cross-sectional specific data.

There was no clear trend of increased or decreased estimates of DFLE for males and females at birth or at age 65 whether using complete or cross-sectional specific data. With a few exceptions, estimates derived from the complete data tended to be slightly higher at birth but slightly lower at age 65 compared with those produced using the cross-sectional specific data. 


\section{Table 3 HLE and DFLE by complete and cross-sectional specific survey data at birth and at age 65 by sex: UK, GB and constituent countries, 2005-2007}

\begin{tabular}{|c|c|c|c|c|c|}
\hline \multicolumn{4}{|l|}{ United Kingdom } & \multicolumn{2}{|r|}{ Years } \\
\hline & & \multicolumn{2}{|c|}{ HLE } & \multicolumn{2}{|c|}{ DFLE } \\
\hline & & Complete & Cross-sectional & Complete & Cross-sectional \\
\hline \multirow[t]{5}{*}{ Males at birth } & UK & 69.2 & 68.4 & 62.6 & 62.5 \\
\hline & GB & 69.2 & 68.5 & 62.6 & 62.6 \\
\hline & England & 69.5 & 68.7 & 62.8 & 62.9 \\
\hline & Wales & 67.7 & 67.1 & 60.3 & 59.3 \\
\hline & Scotland & 68.1 & 67.3 & 62.1 & 61.7 \\
\hline \multirow[t]{5}{*}{ Females at birth } & UK & 71.5 & 70.4 & 63.8 & 64.1 \\
\hline & GB & 71.5 & 70.5 & 63.8 & 63.8 \\
\hline & England & 71.6 & 70.7 & 63.9 & 64.0 \\
\hline & Wales & 70.6 & 69.1 & 63.4 & 63.1 \\
\hline & Scotland & 71.0 & 69.9 & 63.3 & 63.2 \\
\hline \multirow[t]{5}{*}{ Males at age 65} & UK & 13.1 & 12.9 & 9.8 & 10.0 \\
\hline & GB & 13.1 & 12.8 & 9.8 & 10.0 \\
\hline & England & 13.3 & 12.9 & 9.9 & 10.2 \\
\hline & Wales & 12.3 & 12.7 & 9.0 & 8.7 \\
\hline & Scotland & 12.5 & 12.5 & 9.1 & 9.4 \\
\hline \multirow[t]{5}{*}{ Females at age 65} & UK & 15.0 & 14.5 & 10.5 & 10.5 \\
\hline & GB & 15.0 & 14.5 & 10.6 & 10.6 \\
\hline & England & 15.1 & 14.6 & 10.6 & 10.7 \\
\hline & Wales & 14.0 & 13.0 & 10.2 & 9.9 \\
\hline & Scotland & 14.8 & 14.4 & 10.3 & 10.6 \\
\hline
\end{tabular}

\section{Trends in health expectancies}

When comparing the trend over time, estimates of HLE derived from the complete dataset were generally higher than might be expected; while those derived from the cross-sectional specific data were largely comparable with recent trends. Examples of the increases in HLE for males and females at birth and at age 65 in GB are illustrated in figures 1 to 4 . Trends in HLE over time since 2000-2002, including estimates for 2005-2007 based upon both the complete and cross-sectional specific data, are shown. 
Figure 1 HLE for males at birth: by complete and cross-sectional specific data 2000-2002 to 2005-2007

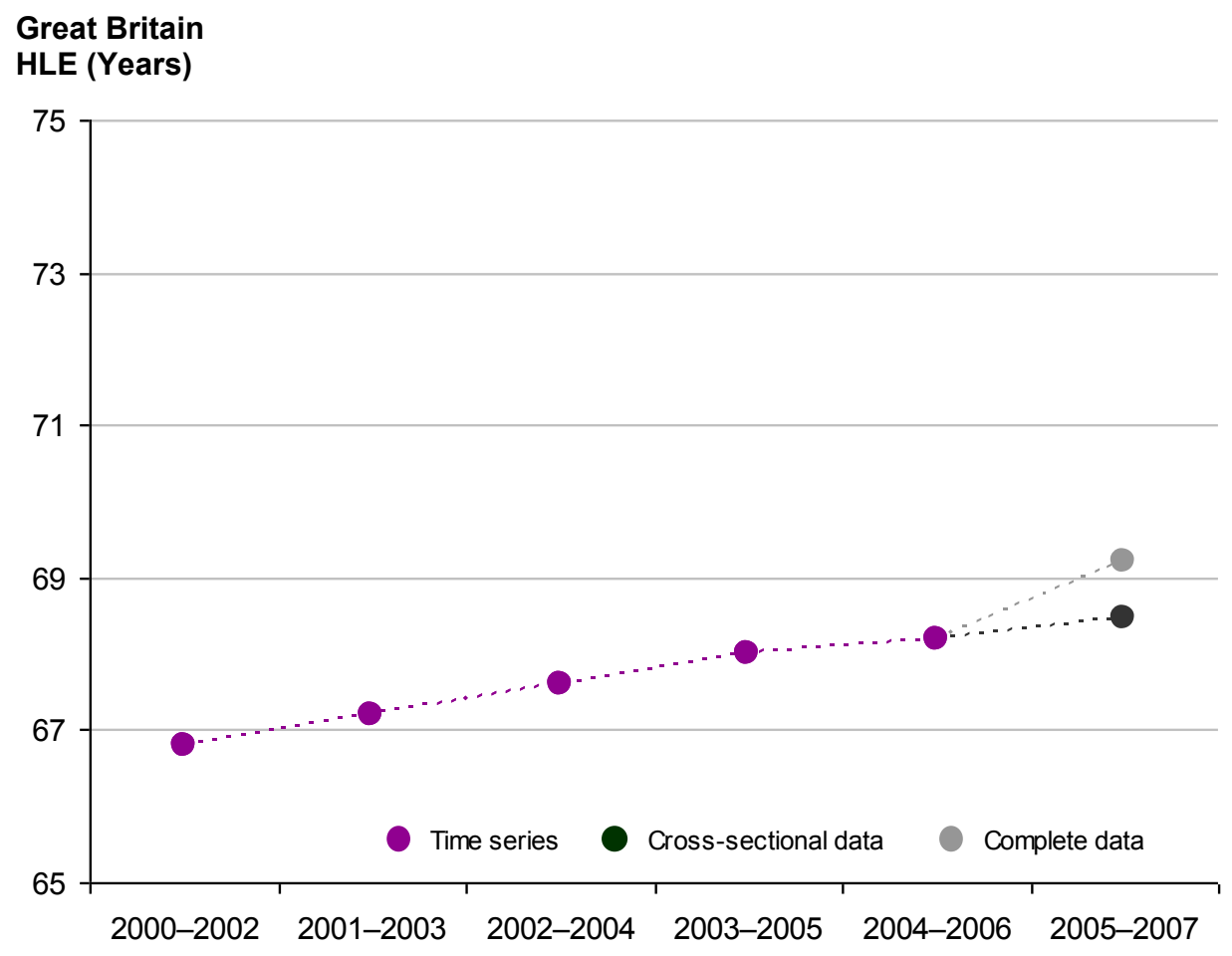

Figure 2 HLE for females at birth: by complete and cross-sectional specific data 2000-2002 to 2005-2007

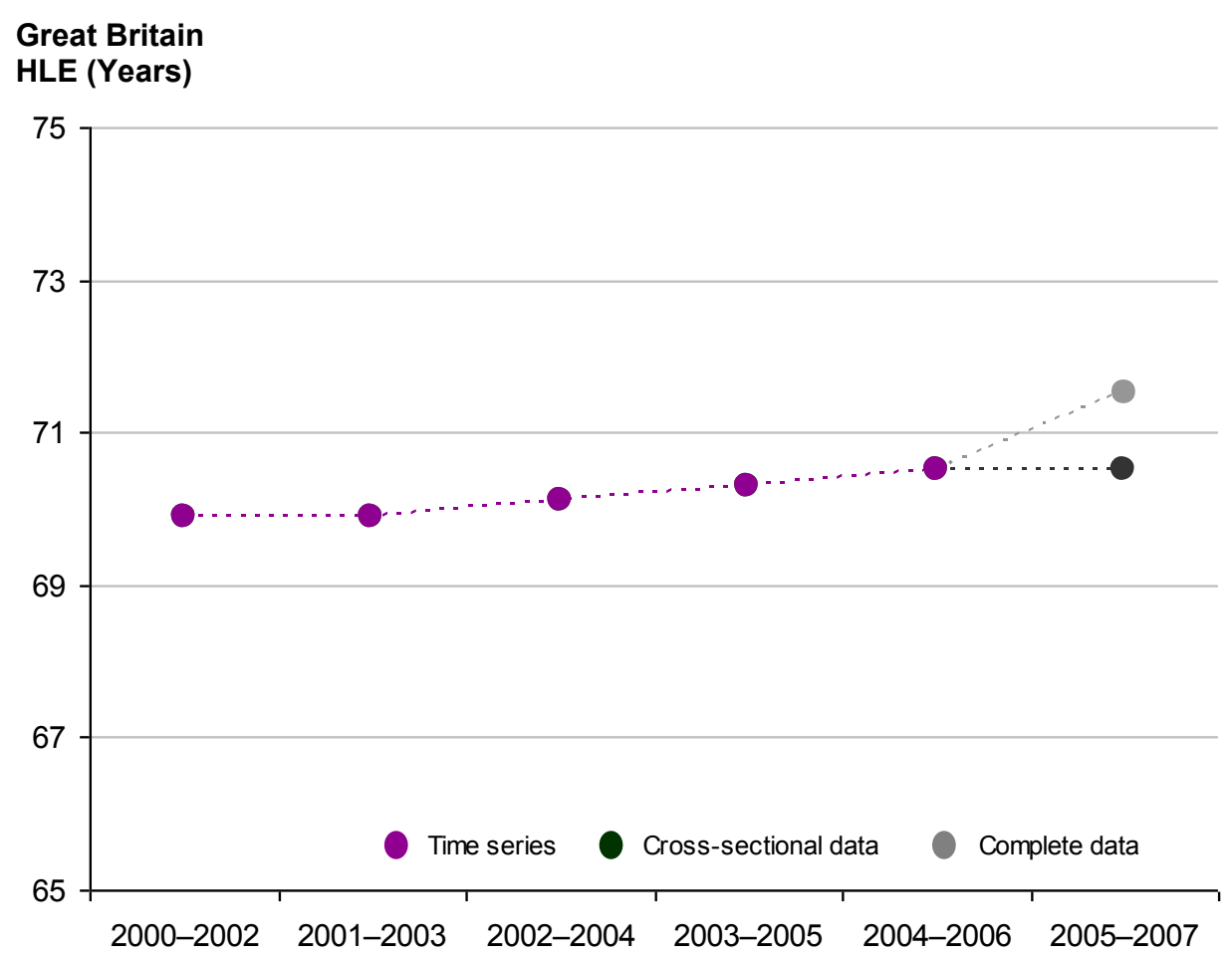


Figure 3 HLE for males at age 65: by complete and cross-sectional specific data 2000-2002 to 2005-2007

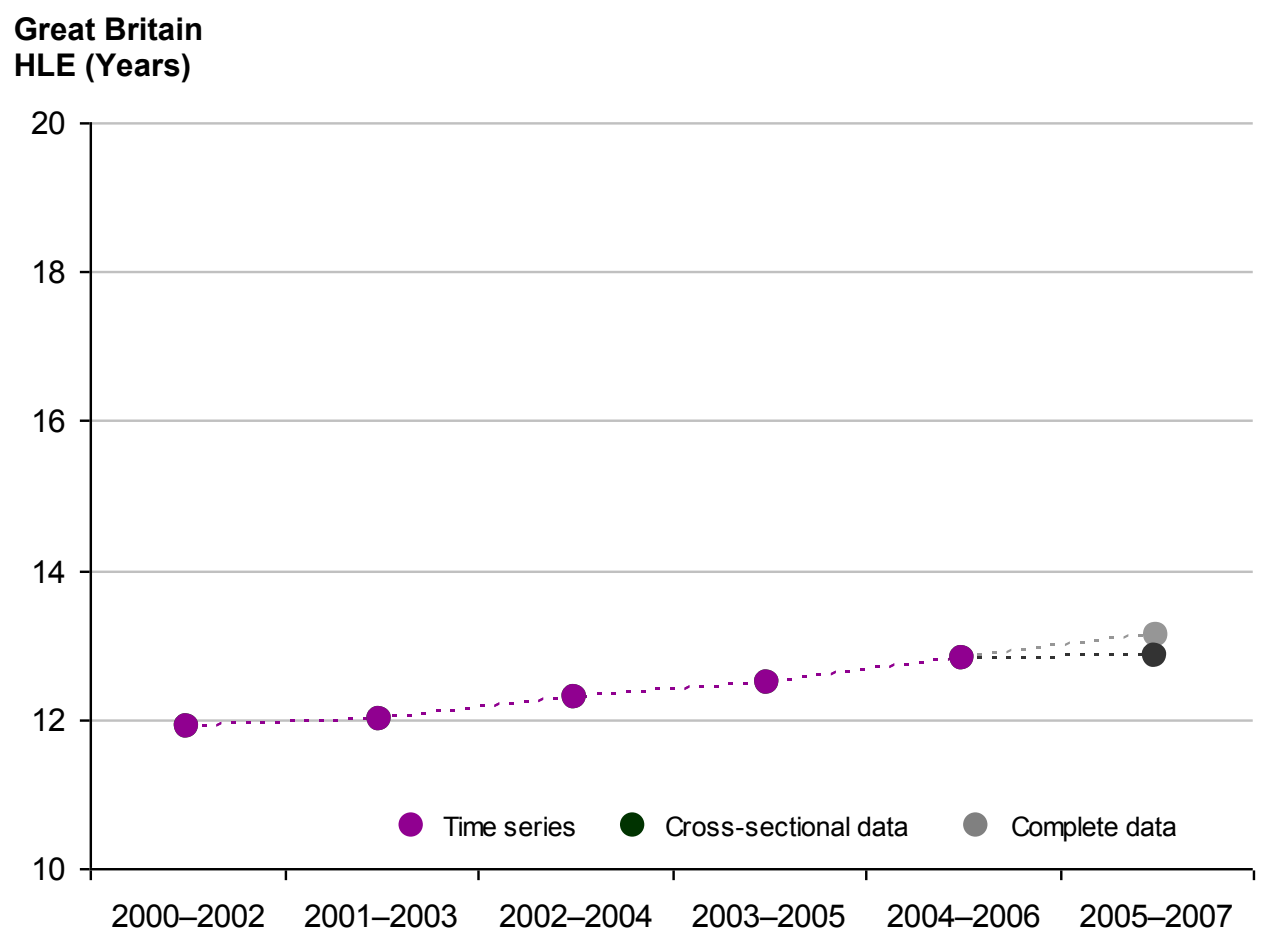

Figure 4 HLE for females at age 65: by complete and cross-sectional specific data 2000-2002 to 2005-2007

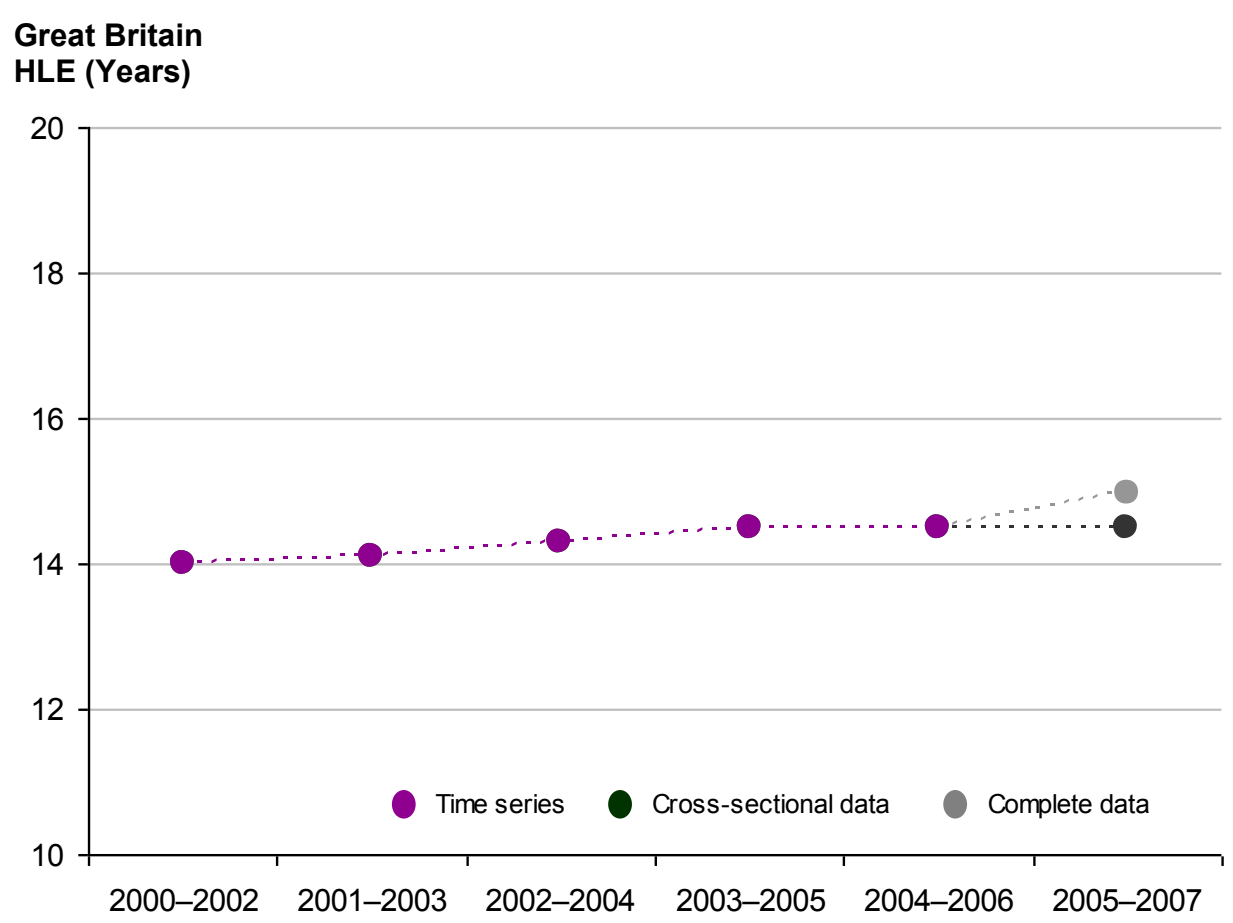


For males at birth in the UK, GB and England there was an average increase in HLE of 0.3 years in each aggregate period from 2000-2002 to 2004-2006 (range 0.2 to 0.5 years). By contrast HLE derived from the complete dataset in 2005-2007 was 1 year higher than in the previous period (2004-2006), twice the largest previous increase between two periods. Estimates of HLE derived from the cross-sectional specific data were within the range of increases in previous years (see Table 4). The relative increase in HLE for females at birth in the UK, GB and England was more pronounced; on average between 2000-2002 and 2004-2006. HLE had increased by 0.1 year (range -0.1 to 0.4 years) for this group, whereas estimates derived from the complete dataset were between 0.9 and 1.1 years higher compared with the previous period. For males and females at birth in Wales and Scotland, HLE derived from the complete data resulted in period increases outside the range seen in previous years, whereas estimates derived from the cross-sectional specific data were within this range (Table 4).

With the exception of Wales, the increase in HLE at age 65 for males was higher when calculated from the complete compared with the cross-sectional data and was at the extreme end of the increases seen over previous periods. For females at age 65, estimates of HLE derived from the complete dataset showed increases beyond the range seen in previous periods in all countries, while those from the cross-sectional specific data were predominantly consistent with previous periods (Table 4).

\section{Application of the sampling design effect of the GHS to the calculation of standard errors}

Incorporating the deff of the GHS into the calculation of standard errors surrounding estimates of HLE and DFLE over the periods 2000-2002 to 2004-2006 resulted in only minor changes to their associated 95 per cent Cls (Table 5).

In most cases the 95 per cent $\mathrm{Cl}$ associated with estimates of HLE and DFLE became slightly wider, reflecting a loss in precision of the estimates overall. At worst the deff resulted in an 8 per cent increase in the width of associated 95 per cent Cls, although improvements of up to 3 per cent were also seen.

On the whole, this change did not unduly affect the ability to detect statistically significant differences between countries or over time. There was, however, a single instance in which the difference between countries was no longer significant using the new Cls: the difference in DFLE for males at birth between England and Scotland in 2001-2003 is no longer statistically significant.

There were also three instances in which significant differences over time were no longer present. For females at age 65 in GB and in England, estimates of HLE in 2004-2006 were no longer significantly different from 2001-2003, and in GB the difference between 2000-2002 and 20032005 estimates was also no longer significant. Comparisons of estimates of HLE and DFLE by aggregated period for the males and females at birth and at age 65 in the UK, Great Britain, England, Wales and Scotland are shown in the Annex, tables A1 to A5. 


\section{Table 4 Comparison of average change in HLE over time with estimates derived from complete and cross-sectional specific datasets}

United Kingdom

Years

\begin{tabular}{|c|c|c|c|c|c|}
\hline & & \multicolumn{2}{|c|}{ Time series } & \multirow{2}{*}{$\begin{array}{r}\text { Complete } \\
\text { Change between } \\
2004-2006 \text { and } \\
2005-2007\end{array}$} & \multirow{2}{*}{$\begin{array}{r}\text { Cross-sectional } \\
\text { Change between } \\
2004-2006 \text { anc } \\
2005-2007\end{array}$} \\
\hline & & $\begin{array}{r}\text { Average change } \\
\text { between 2000- } \\
2002 \text { and 2004- } \\
2006\end{array}$ & Range & & \\
\hline \multirow[t]{5}{*}{ Males at birth } & UK & 0.3 & 0.3 to 0.5 & 1.0 & 0.2 \\
\hline & GB & 0.3 & 0.2 to 0.4 & 1.0 & 0.3 \\
\hline & England & 0.3 & 0.2 to 0.4 & 1.0 & 0.2 \\
\hline & Wales & 0.2 & -0.4 to 0.4 & 1.0 & 0.4 \\
\hline & Scotland & 0.2 & -0.5 to 0.9 & 1.6 & 0.8 \\
\hline \multirow[t]{5}{*}{ Females at birth } & UK & 0.1 & 0.0 to 0.2 & 1.1 & 0.0 \\
\hline & GB & 0.1 & 0.0 to 0.3 & 1.0 & 0.0 \\
\hline & England & 0.1 & -0.1 to 0.4 & 0.9 & 0.0 \\
\hline & Wales & -0.1 & -0.5 to 0.6 & 1.7 & 0.2 \\
\hline & Scotland & 0.2 & -0.1 to 0.8 & 1.4 & 0.3 \\
\hline \multirow[t]{5}{*}{ Males at age 65} & UK & 0.2 & 0.1 to 0.3 & 0.3 & 0.1 \\
\hline & GB & 0.2 & 0.1 to 0.3 & 0.3 & 0.0 \\
\hline & England & 0.2 & 0.1 to 0.4 & 0.4 & 0.0 \\
\hline & Wales & 0.2 & -0.4 to 0.9 & 0.0 & 0.4 \\
\hline & Scotland & 0.1 & -0.2 to 0.7 & 0.3 & 0.3 \\
\hline \multirow[t]{5}{*}{ Females at age 65} & UK & 0.1 & 0.0 to 0.3 & 0.5 & 0.0 \\
\hline & GB & 0.1 & 0.0 to 0.2 & 0.5 & 0.0 \\
\hline & England & 0.1 & -0.1 to 0.3 & 0.4 & -0.1 \\
\hline & Wales & 0.1 & -0.3 to 0.4 & 0.7 & -0.3 \\
\hline & Scotland & 0.1 & -0.1 to 0.4 & 0.6 & 0.2 \\
\hline
\end{tabular}




\section{Table $5 \quad$ Average precision of estimates of HLE and DFLE at birth and at age 65 by sex in original and deff adjusted 95 per cent $\mathrm{Cl}$ : UK, GB and constituent countries 2000-2002 to 2004-2006}

\begin{tabular}{|c|c|c|c|c|c|c|c|}
\hline \multicolumn{2}{|l|}{ United Kingdom } & & & & \multicolumn{3}{|r|}{ Years } \\
\hline & & \multicolumn{3}{|c|}{ HLE } & \multicolumn{3}{|c|}{ DFLE } \\
\hline & & $\begin{array}{r}\text { Original } 95 \\
\text { per cent } \\
\mathrm{Cl}\end{array}$ & $\begin{array}{r}\text { deff adjusted } \\
95 \text { per cent } \\
\mathrm{Cl}\end{array}$ & $\begin{array}{l}\text { Change in } \\
\text { width of } \mathrm{Cl} \\
\text { (per cent) }\end{array}$ & $\begin{array}{r}\text { Original } 95 \\
\text { per cent } \\
\mathrm{Cl}\end{array}$ & $\begin{array}{r}\text { deff adjusted } \\
95 \text { per cent } \\
\mathrm{Cl}\end{array}$ & $\begin{array}{l}\text { Change in } \\
\text { width of } \mathrm{Cl} \\
\text { (per cent) }\end{array}$ \\
\hline \multirow[t]{5}{*}{ Males at birth } & UK & 0.5 & 0.5 & -3.0 & 0.6 & 0.6 & -0.7 \\
\hline & GB & 0.6 & 0.6 & -4.0 & 0.7 & 0.7 & -3.1 \\
\hline & England & 0.6 & 0.6 & -3.1 & 0.7 & 0.7 & -3.0 \\
\hline & Wales & 2.6 & 2.7 & -2.7 & 2.9 & 3.0 & -3.1 \\
\hline & Scotland & 1.8 & 1.9 & -5.5 & 2.1 & 2.1 & -2.2 \\
\hline \multirow[t]{5}{*}{ Females at birth } & UK & 0.5 & 0.5 & -3.4 & 0.6 & 0.6 & -1.7 \\
\hline & GB & 0.6 & 0.6 & -4.4 & 0.7 & 0.7 & -4.7 \\
\hline & England & 0.7 & 0.7 & -4.0 & 0.7 & 0.8 & -4.6 \\
\hline & Wales & 2.7 & 2.7 & -2.1 & 3.0 & 3.0 & -2.5 \\
\hline & Scotland & 1.8 & 1.9 & -4.7 & 2.2 & 2.2 & -3.3 \\
\hline \multirow[t]{5}{*}{ Males at age 65} & UK & 0.4 & 0.4 & 0.6 & 0.4 & 0.4 & 3.2 \\
\hline & GB & 0.4 & 0.4 & 0.7 & 0.5 & 0.5 & 2.4 \\
\hline & England & 0.4 & 0.4 & 1.8 & 0.5 & 0.5 & 2.8 \\
\hline & Wales & 1.8 & 1.9 & -1.4 & 1.9 & 2.0 & -0.5 \\
\hline & Scotland & 1.3 & 1.4 & -1.6 & 1.5 & 1.5 & 3.3 \\
\hline \multirow[t]{5}{*}{ Females at age 65} & UK & 0.4 & 0.4 & -4.1 & 0.4 & 0.4 & -1.9 \\
\hline & GB & 0.4 & 0.5 & -5.2 & 0.5 & 0.5 & -4.4 \\
\hline & England & 0.5 & 0.5 & -4.5 & 0.5 & 0.6 & -4.7 \\
\hline & Wales & 1.9 & 1.9 & -2.2 & 2.0 & 2.0 & -0.5 \\
\hline & Scotland & 1.3 & 1.4 & -8.2 & 1.5 & 1.6 & -5.8 \\
\hline
\end{tabular}

\section{Discussion}

The transition of the GHS from a cross-sectional to a rotating-panel longitudinal design in 2005 has an effect on the continuity of the ONS HE time series, established in 1981 for GB and in 2001 for the UK.

The prevalence of 'Good' or 'Fairly Good' health was significantly higher among respondents in the longitudinal compared with the cross-sectional elements of the survey in 2006 and 2007, indicating a selective loss or attrition of those in the poorest health from the sample. Interestingly, there were 
no differences between the cross-sectional and longitudinal elements of the survey in terms of limiting long-standing illness in any period examined. In part, the stability of this measure, compared with general health, is due to the fact that it is on the whole more prevalent than 'Not Good' health; people with a limiting long-standing illness may nevertheless report their general health state as 'Good' or 'Fairly Good'. ${ }^{4}$ The selective loss or attrition of people in the poorest health from the longitudinal element of the survey will therefore have less of an impact on the overall prevalence of limiting long-standing illness than on general health states.

The prevalence of 'Good' and 'Fairly Good' health remained higher when longitudinal and crosssectional data were combined to produce a complete 2005-2007 dataset, compared with crosssectional data alone over this period. Estimates of HLE calculated from the complete dataset were higher for males and females at birth in the UK, GB and England than those calculated from the cross-sectional specific data. The period increase in HLE according to the complete data was also implausibly higher than that of recent years, indicating that these data will exaggerate the expected years of life spent in 'Good' or 'Fairly Good' health, particularly at birth.

Although generally higher, the differences in the prevalence of limiting long-standing illness and in estimates of DFLE for males or females at birth or at age 65 using the complete data were comparable to those estimated using cross-sectional specific data.

Incorporating the longitudinal element of the $\mathrm{GHS}(\mathrm{L})$ into calculations of $\mathrm{HE}$ leads to a deviation in estimated HLE compared with recent trends, whereas, estimates calculated from the crosssectional specific data are broadly in line with increases seen in recent years. The continued use of the cross-sectional survey data alone, excluding the longitudinal component, therefore, is advisable to maintain continuity of the ONS HE time series. This approach, however, decreases the precision of HE estimates.

This report does not include measures of the statistical significance of differences in HE between the complete and cross-sectional specific $\mathrm{GHS}(\mathrm{L})$ elements. It is likely that the covariation of health states between successive interviews in the longitudinal panels will have an effect on the size of SEs and associated 95 per cent Cls of HE estimates in the complete 2005-2007 data. The methodology to calculate SEs is, at the time of writing, under development and will be reported in a future article.

Models comparing estimates of HLE and DFLE based upon the exclusion of two-thirds of the GB survey sample over two or three years; equivalent to the size of the cross-sectional panel of the GHS(L) survey in 2005-2007 and 2006-2008 respectively, with estimates based on GHS sample sizes seen prior to 2004-2006, suggest that the precision of estimates will decline with falling sample sizes, (data not shown). On average it appears that the 95 per cent $\mathrm{Cl}$ associated with estimates of HLE and DFLE for males and females at birth and at age 65 will increase in width by 15 to 34 per cent with two years of reduced data and 47 to 87 per cent with three years of reduced data. Although these increases seem large in terms of percentage increase, they equate at worst to increases in the width of 95 per cent Cls of less than 1 year at birth and less than 0.5 years at age 65 . However, this loss of precision is likely to impair our ability to identify real differences in HE between countries or over time. 
Since 2000-2002 the question designed to capture the prevalence of general health in the population has remained unchanged. However, estimates of HLE based on this question appear for the last time in 2005-2007: it has been replaced by a broader EU-harmonised general health question since $2008 .^{4}$

As with DFLE, estimates of HLE based upon this new question will be less affected by the inclusion of longitudinal data in the GHS(L) in 2005-2007 (data not shown) because of the stronger relationship between states of 'Good' health derived from this question and the presence of a limiting long-standing illness. Therefore, the inclusion of the longitudinal $\mathrm{GHS}(\mathrm{L})$ panels will be considered again for HE estimates in 2006-2008 once the methodology to calculate the SE of the complete dataset has been developed and can be compared more accurately with the crosssectional survey data from this period.

\section{Conclusion}

The transition of the GHS(L) from a cross-sectional to a rotating-panel longitudinal design in 2005 leads to increases in the estimated population prevalence of 'Good' and 'Fairly Good' health states and, therefore estimates of HLE that depart implausibly from the established time trend.

Using the GHS $(\mathrm{L})$ cross-sectional specific data for estimating HE for the period 2005-2007 appears to represent the best way to maintain the continuity of this important series. With further methodological development and the replacement of the question traditionally used to calculate the prevalence of 'Good' or 'Fairly Good' health, it is conceivable that ONS will adopt the complete GHS(L) (now General Lifestyle module) datasets for the reporting of HE in the period 2006-2008. This is likely to avoid the loss of precision seen with the reduction in the survey sample when using only the cross-sectional panels.

ONS has improved the method to calculate the SE and associated 95 per cent $\mathrm{Cl}$ of estimates of $\mathrm{HE}$ by incorporating the design effect of the $\mathrm{GHS}(\mathrm{L})$. This change has a negligible impact in the short term but ultimately improves the measured precision of these estimates. 


\section{Acknowledgements}

We are grateful for the support of Robert Bucknall and Karl Ashworth of ONS Sample Design and Estimation Unit in the development of this article.

\section{References}

1. Lynn P (2009) Methodology of Longitudinal Surveys Wiley, West Sussex.

2. Jagger C (1999) Health Expectancy Calculation by the Sullivan Method: A Practical Guide, NUPRI Research Paper Series No 68, Tokyo.

3. Breakwell C and Bajekal M (2005) 'Review of sources and methods to monitor healthy life expectancy', Health Statistics Quarterly 26, 17-22. Available on the Office for National Statistics website at: www.statistics.gov.uk/cci/article.asp?ID=1146\&Pos=1\&ColRank=1\&Rank=224

4. Smith MP and White C (2008) 'An investigation of the impact of question change on estimates of general health prevalence and healthy life expectancy'. Health Statistics Quarterly 41, 28-42. Available on the Office for National Statistics website at: www.statistics.gov.uk/cci/article. $a s p ? \mid \mathrm{D}=2140 \& \mathrm{Pos}=1 \&$ ColRank=1\&Rank=1 